\title{
Prototipo Para la ORIENTACión AUTOMÁTICA DE PANELES SOLARES
}

\author{
Prototype for Automatic Orientation \\ of Solar Panels
}

\section{${ }^{1}$ Erika Natalia Abello Mendoza ${ }^{2}$ William Fernando Bernal Suarez}

\author{
Fundación Universitaria Juan de Castellanos \\ Facultad de Ingeniería y Ciencias Básicas \\ ${ }^{1}$ eabelloajdc.edu.co, orcid.or/0000-0002-4042-5041 \\ ${ }^{2}$ wbernalejdc.edu.co, orcid.org/0000-0001-9901-2076
}

Recibido: 14/09/2016 Aprobado: 30/10/2016

\section{RESUMEN}

En este artículo se describe el proceso de diseño e implementación de un prototipo para la orientación automática de paneles solares, aplicando áreas del conocimiento de la electrónica, control y energías renovables; esto con fines netamente académicos en el análisis y mejoramiento de la eficiencia de sistemas fotovoltaicos. Explicando bloque por bloque el proceso de adquisición de señales y control, empleando procesos de calibración y patronaje que aseguren una confiabilidad adecuada del prototipo con luxómetros y equipos de laboratorio como osciloscopio, generador de señales, entre otros.

Palabras clave: arduino, luxómetro, LDR orientador solar.

\begin{abstract}
This article describes the process of designing and implementing of a prototype for automatic orientation of solar panels, applying the areas of electronic knowledge, control and renewable energies; this for academic purposes in the analysis and improvement of photovoltaic systems efficiency. Explaining block by block the signal and control acquisition process, applying the calibration and the pattern processes, that assure an adequate reliability of the prototype with luxometers and laboratory equipment, like oscilloscope and signals generator.
\end{abstract}

Keywords: arduino, luxometer, solar advisor. 


\section{INTRODUCCIÓN}

El creciente desarrollo industrial ha traído como consecuencia un incremento importante del consumo de electricidad generada por combustibles fósiles, caracterizándose éstos por ser recursos limitados y provocando un impacto negativo en el medio ambiente. Para ello, se plantea el uso de energías alternativas, que disponen del aprovechamiento de fuentes naturales y ayudan a la reducción de emisiones de $\mathrm{CO} 2$. Este asunto es tratado en diferentes países, un ejemplo es Estados Unidos (Brown 2015).

Una de las alternativas es la energía solar la cual se puede convertir directamente en energía eléctrica mediante el empleo de celdas solares fotovoltaicas (Beltrán Adán 2007) y las cuales en la región de Boyacá presentan una alta viabilidad para su uso.

En varios municipios de Colombia, es importante contar con una red de estaciones para el monitoreo de las condiciones atmosféricas (López Jiménez 2014). Dicho esto, el Instituto de Hidrología, Meteorología y Estudios Ambientales (IDEAM) junto con la unidad de planeación Minero Energética (Upme), como entidades nacionales encargadas del planteamiento energético y de la medición de variables ambientales, cuentan con estaciones meteorológicas ubicadas en distintos puntos del país, de esta manera, se logra obtener mapas de radiación solar global (Upme 2014).

En Colombia existe una alta radiación solar dependiendo de la región, por ejemplo, en la zona Andina sobresale el altiplano Cundiboyacense, en el cual los valores máximos se registran en el mes febrero con niveles de $480 \mathrm{cal} / \mathrm{cm} 2 /$ día $(232.32 \mathrm{~W} / \mathrm{m} 2)$, descendiendo gradualmente hasta junio, con 420 $\mathrm{cal} / \mathrm{cm} 2 /$ día $(203.28 \mathrm{~W} / \mathrm{m} 2)$ y nuevamente se incrementan poco a poco, como se observa en la Fig. 1 (Ideam 2014).

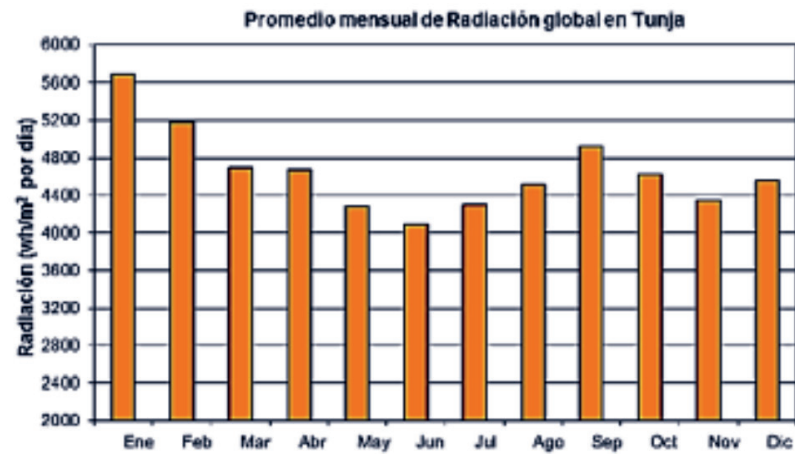

Fig. 1 Datos de radiación solar en Tunja Fuente: IDEAM disponible en http://atlas.ideam.gov.co/visorAtlasRadiacion.html

De la misma forma los datos obtenidos de la estación meteorológica código:24035130, ubicada en la Uptc seccional Tunja (Ideam 2016), determinan que la ciudad de Tunja cuenta con aproximadamente 13.000 $\mathrm{cal} / \mathrm{cm} 2(6200 \mathrm{~W} / \mathrm{m} 2)$ cada mes, es decir, que se trata de un lugar estratégico para el uso de la energía solar.

La dificultad que se presenta con el uso de este recurso radica en que los sistemas fotovoltaicos (Guerrero Pérez 2013) se ensamblan en estructuras fijas, haciendo que los paneles solares no tengan la capacidad de aprovechar óptimamente la energía solar generada durante todo el día, ya que la radiación varía con la posición del sol.

Hoy en día existen herramientas que permiten hacer estos procesos de forma automática para la orientación paralela del panel continuamente al sol, como es el caso los orientadores solares, diseńados para adquirir la energía del sol de acuerdo a su movimiento diario (Machado et al. 2015).

El objetivo de este proyecto fue implementar un prototipo automático para el seguimiento solar de dos ejes de inclinación (Guardado \& Rivera 2012), usando sensores tipo LDR que medirán la intensidad lumínica y permitirán al sistema de control referenciarse para posicionar de forma paralela el panel solar respecto a la posición del sol, permitiendo un máximo aprovechamiento de este, y asegurando la obtención de los mejores niveles de energía del panel fotovoltaico. 


\section{Desarrollo de Contenidos}

\section{A. Metodología}

En la Fig. 2 se observa el proceso para la construcción del prototipo. El primer paso es la investigación teórica de paneles solares, estructuras móviles y antecedentes, así como los materiales a usar tales como sensores, actuadores, componentes y elementos electrónicos.

El segundo paso, es el diseño de la estructura desarrollado con el software Autodesk Inventor ${ }^{\bullet}$, para ello se realizaron pruebas de adaptación con los parámetros establecidos. Esto, basados en la estructura previa del código mediante un diagrama de flujo.

El tercer paso es la construcción del prototipo, ajustándose a los requerimientos técnicos y funcionales de disposición de elementos electrónicos y a las dimensiones del panel solar fotovoltaico, para finalmente efectuar las pruebas de campo, evaluar la fiabilidad del sistema $\mathrm{y}$, proceder a hacer las tomas de datos comparativas respecto a paneles estáticos.

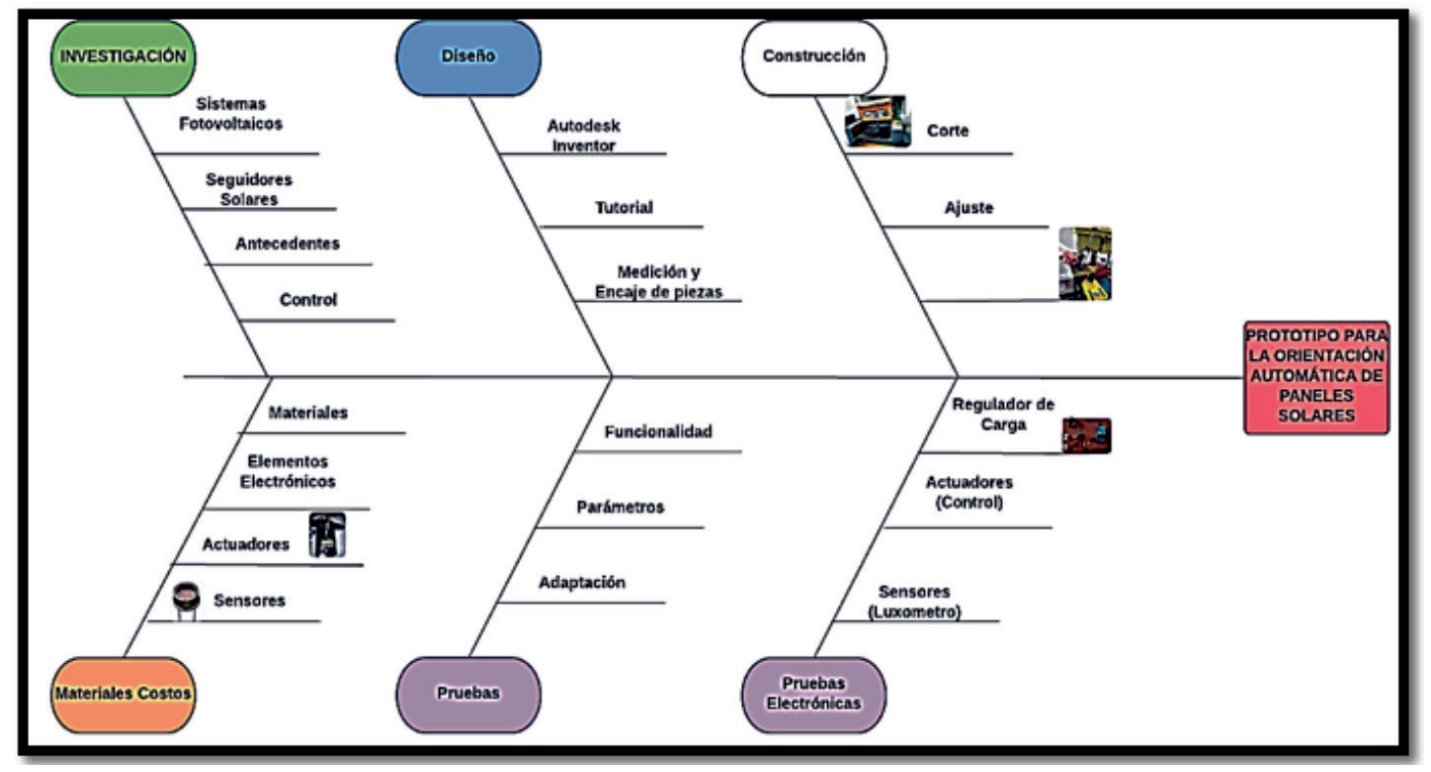

Fig. 2 Metodología utilizada para el desarrollo del prototipo.

Fuente: autores

\section{B. Diagrama de bloques}

En la Fig. 3 se describe el proceso del sistema del prototipo. Los tres primeros bloques del diagrama muestran la alimentación del sistema, los cuales son: Panel Solar (1), se encarga de recibir la luz solar para cargar la batería (3), antes de este proceso se pasa por el regulador de carga (2), siendo necesario para realizar un correcto proceso de carga de la batería y su protección, estabilizar la energía y regular el voltaje para alimentar la placa electrónica y el arduino.
Los siguientes bloques hacen referencia al controlador del prototipo, a partir de la programación en una tarjeta Arduino Uno (4), donde los sensores LDR (5) se encargan de enviar la señal que determinará en el sistema de control los movimientos y posición final de los servomotores (6) para una orientación paralela al sol. Finalmente, los datos de los sensores (7) se almacenan en una tarjeta $S d$ (secure digital) de la placa Arduino Shield, lo cual hace posible que el usuario final descargue dichos datos para ser analizados. 


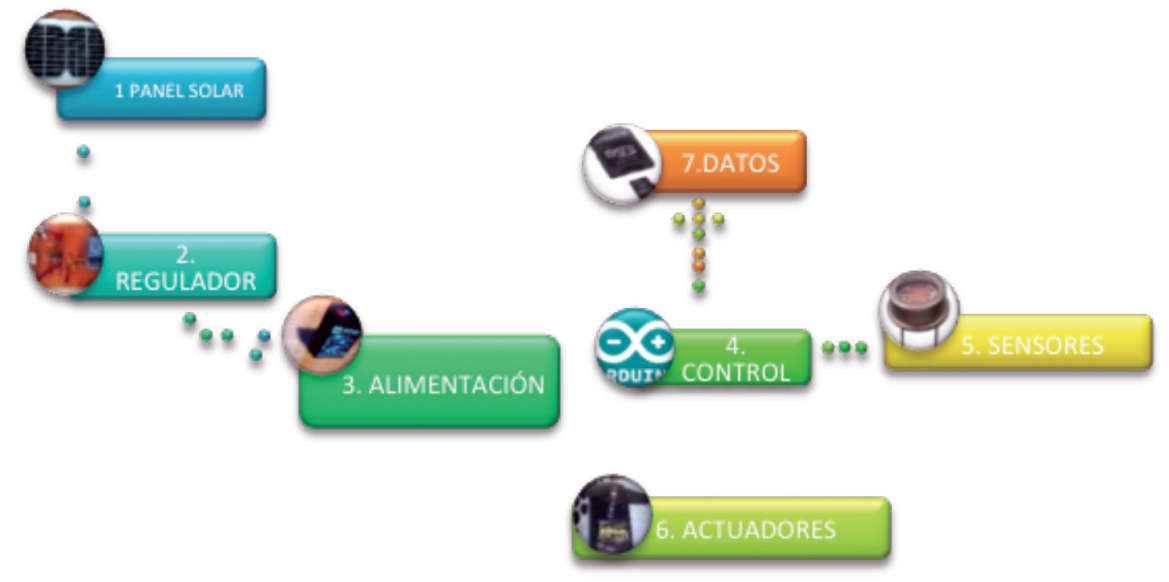

Fuente: autores

Fig. 3 Diagrama de bloques del prototipo.

\section{1) Panel Solar}

El panel solar utilizado en este proyecto es un panel de $5 \mathrm{~W}$, ya que las necesidades energéticas del prototipo no requieren de un panel de mayor vatiaje y cumplirá dos tareas específicas, la primera cargar la batería que alimenta al sistema y la segunda permitir identificar la energía eléctrica que este va generando según la posición en la que este se encuentre y que estará definida por la estructura móvil de orientación automática. En la siguiente tabla, apreciamos sus especificaciones técnicas.

\section{TABla 1.}

Especificaciones del panel solar

\begin{tabular}{|l|c|}
\hline \multicolumn{2}{|c|}{ Especificaciones } \\
\hline Máximo poder & M5 \\
\hline Mínimo poder de voltaje & $5 \mathrm{~W}+-3 \%$ \\
\hline Máximo poder de corriente & $17.5 \mathrm{~V}$ \\
\hline Voltaje en circuito abierto (Voc) & $0.29^{\mathrm{a}}$ \\
\hline Corriente de corto circuito (Isc) & $0.32^{\mathrm{a}}$ \\
\hline Tensión máxima del sistema & $\mathrm{DC} 1000 \mathrm{~V}$ \\
\hline Máximo fusible de potencia & $5^{\mathrm{a}}$ \\
\hline STC (Condición de prueba estándar) & AM1.5 $1000 \mathrm{~W} / \mathrm{m} 2$ \\
$25^{\circ} \mathrm{C}$
\end{tabular}

\section{2) Regulador de carga}

El regulador de carga controla el nivel de carga de la batería, protegiéndola de descargas y sobrecargas que puedan dañarla. En la Fig. 4, se observa el diagrama esquemático del regulador montado en este sistema, cuya finalidad principal va de la mano con los niveles de corriente suministrados por el panel solar. Es importante aclarar que se aplicó la calibración del regulador y que este proceso cambia según las necesidades o características de la batería.

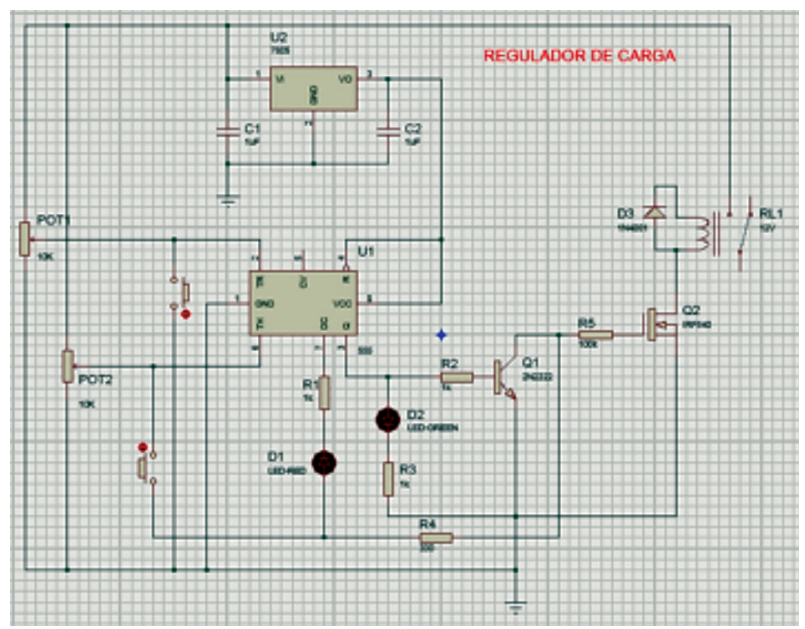

Fig. 4 Esquema del regulador de carga

\section{3) Programa de control}

Para crear el movimiento del panel solar, se trabajó en la plataforma de programación de Arduino 1.0.3, donde 
se estructuró el código general con el manejo de las librerías correspondientes de sensores y servomotores y así obtener la respuesta esperada del sistema. En la figura 5, se observa el diagrama de flujo del sistema de control; Se declararon las variables necesarias como sensores (LDR) y actuadores (servomotores) y, la estructura secuencial lógica según las lecturas obtenidas por las 4 LDR para así determinar la respuesta (orientación) más adecuada de los servomotores.

Es importante comparar los valores máximos de luminosidad para poder determinar la posición más adecuada en la cual se deben ubicar los servomotores, para esto se definieron las siguientes posiciones $\mathrm{ArD}$ (arriba derecha), AbI (abajo izquierda), ArI (arriba izquierda) y AbD (abajo derecha). Según los cambios de luminosidad en las fotorresistencias, se definió un esquema comparativo de la siguiente forma:

Si ArD > AbI el servomotor se posiciona en el rango máximo, de lo contrario gira a la izquierda, así mismo si ArI $>$ A bD el servomotor gira a la izquierda de lo contrario a la derecha, y así sucesivamente según corresponda.

Toda esta información puede ser visualizada por medio del monitor serial de Arduino, esto con el fin de observar en tiempo real la lectura y señal de posición enviada a los servomotores.

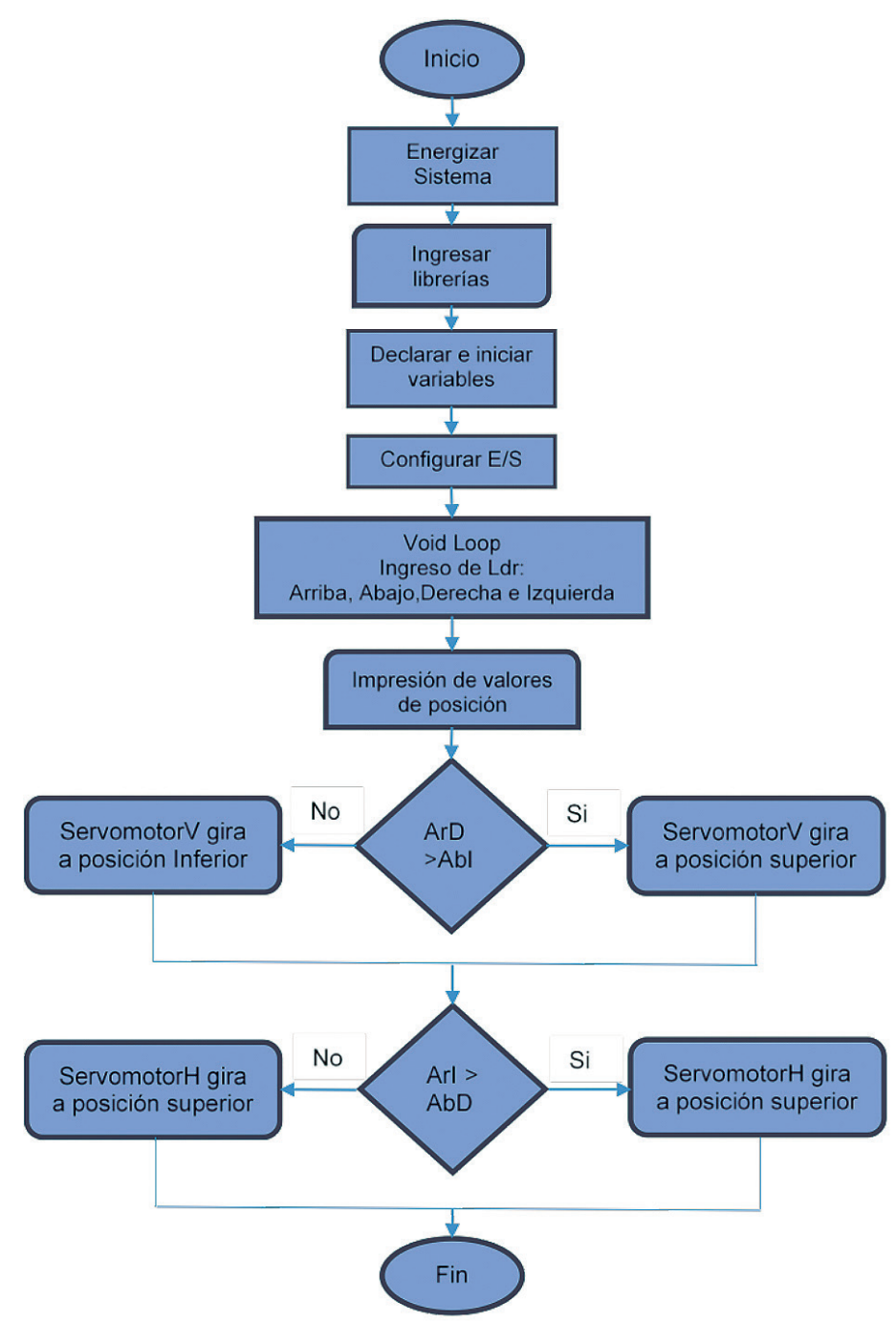

Fig. 5 Diagrama de flujo del programa de control. 


\section{4) Sensores}

Se determinó usar sensores de luminosidad (Herrera \& Molina 2010), en este caso cuatro fotorresistencias o LDR, para obtener los niveles de intensidad lumínica incidentes en el panel. de este modo, y previo acondicionamiento y calibración normalizar esta señal para ser leída adecuadamente por el microcontrolador. En la figura 6 se puede observar el acondicionamiento del nivel de tensión por medio de un divisor de voltaje.

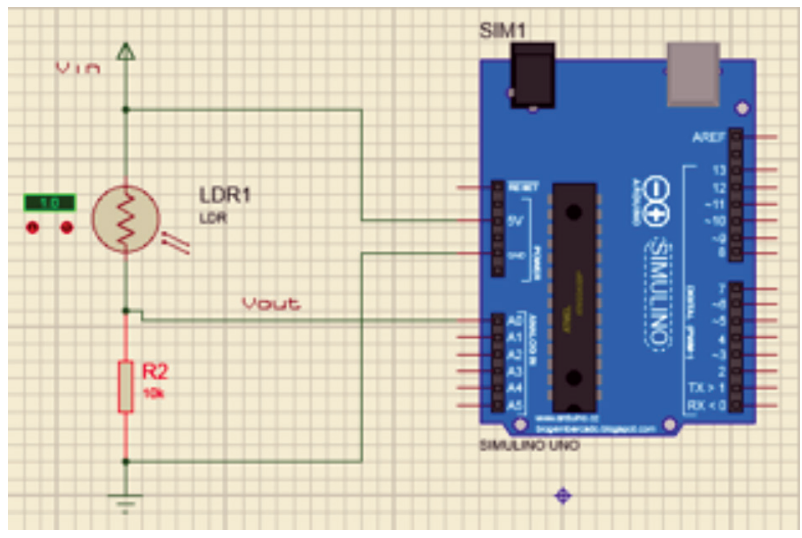

Fig. 6 Divisor de voltaje

\section{5) Servomotores}

Para la correcta ubicación del panel y dadas las necesidades requeridas en cuanto a velocidad de respuesta y precisión, se estableció que el uso de dos servomotores era el que mejor se adecuaba a este sistema, en Fig. 7, se aprecia el servo usado, el cual cuenta con un torque de $12 \mathrm{~kg} / \mathrm{cm}$ y piñonera metálica, ajustándose de forma óptima para la ejecución del movimiento de la estructura de dos grados de libertad.

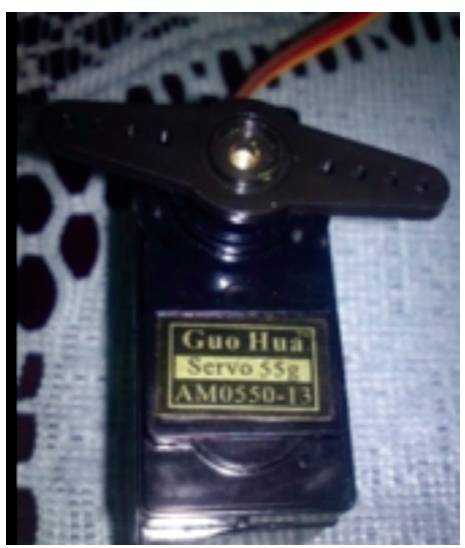

Fig. 7 Servomotor Guo Hua.

\section{6) Calibración $L D R$}

Teniendo en cuenta que la resistencia óhmica de la LDR varía en función de la cantidad de luz que incide sobre ésta, es decir, el valor de la resistencia es inversamente proporcional al incremento o disminución de la luz, se procedió a calibrar las LDR de la siguiente manera; con un multímetro se tomaron datos de la resistencia óhmica de cada una de ellas aproximadamente cada minuto, y paralelamente se midió la luminosidad con un luxómetro, en este caso se utilizó el Amprobe LM120 como se puede apreciar en la Fig. 8.
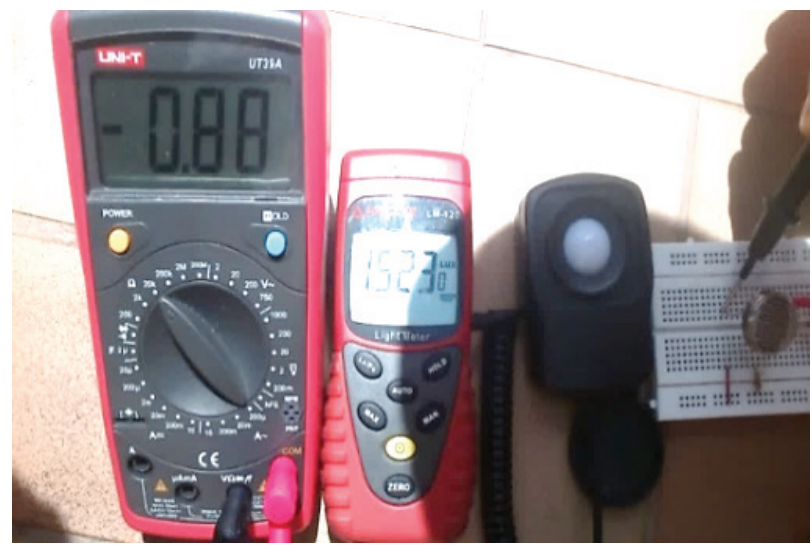

Fig. 8 Calibración LDR con luxómetro

Utilizando el programa Microsoft Excel $^{\circledR}$ como herramienta de análisis de datos (López, Martínez y Almario 2016), se determinó que dicha filiación se convierte en lineal si se utiliza escala logarítmica, representada en la Fig. 9.

\section{Calibración LDR}

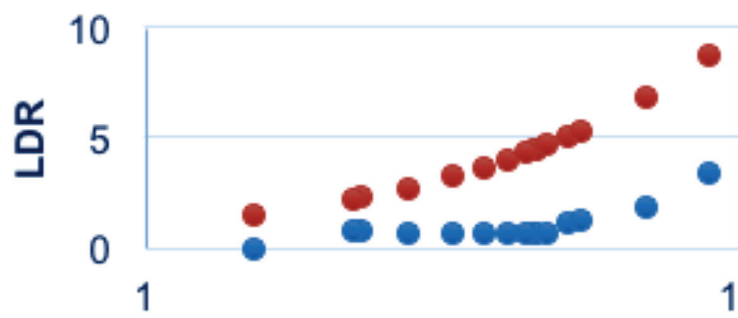

Luxometro

-LDR - R $(\mathrm{k} \Omega) \quad \bullet$ Luxometro - L(Lux)

Fig. 9 comparación LDR y Luxómetro 


\section{7) Caja de Control}

En la Fig. 10 se muestra el diagrama de bloques de la caja de control, siendo la base del prototipo.

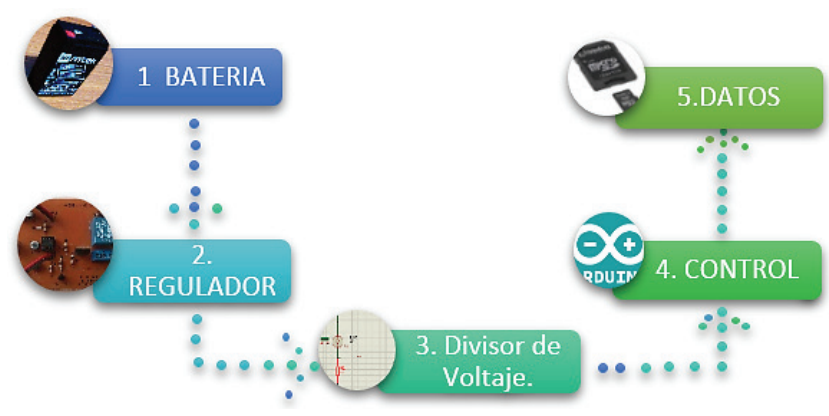

Fig. 10 Diagrama de bloques de la caja de control.

Las partes de la caja de control son:

1. Batería

2. Regulador de carga conectado al panel solar y a la batería.

3. Se encuentra el diodo de bloqueo para el panel solar, el divisor de voltaje para las LDR y un regulador para proporcionar los $5 \mathrm{~V}$ de alimentación para el Arduino, los servomotores y las fotorresistencias.

4. Bloque de Control compuesto por la placa de Arduino Uno, conectado a los servomotores y sensores.

5. Ethernet Shield incorporado en la placa, permitiendo almacenar los datos de los sensores LDR.

\section{Resultados}

\section{Calibración $L D R$}

En el proceso de calibración, en la cual se evidenció la dependencia entre resistencia e iluminación se representa con la fórmula (Pallás Areny 2003):

$$
\mathbf{R}=\mathbf{A} \cdot \mathbf{L}-\boldsymbol{\alpha}
$$

Donde $R$ es la resistencia en ohmios; $L$ es la iluminación en lux; A $y \alpha$ son constantes, estas dependen del tipo de material con que se ha construido la fotorresistencia y del proceso de manufactura.

De acuerdo a los resultados en tiempo real, se observa que los datos de la resistencia óhmica de las LDR no logran ser completamente lineales con los datos obtenidos del luxómetro. Dicho esto, se determinó que los valores comparables de los sensores no son confiables para determinar una relación de luminosidad como se puede apreciar en la Fig. 9.

\section{Prototipo Final}

El ensamble final de las piezas diseñadas, desarrollado con base a la estructura inferior de un brazo robótico, da como resultado el prototipo para la orientación automática de paneles solares de dos ejes, el cual tiene un movimiento de Este a Oeste y de manera circular. Además, es importante el estudio de la dinámica del consumo de energía en el dispositivo, con el fin de buscar una gestión óptima del recurso energético (Amarillo \& Trujillo 2015) Fig. 11.

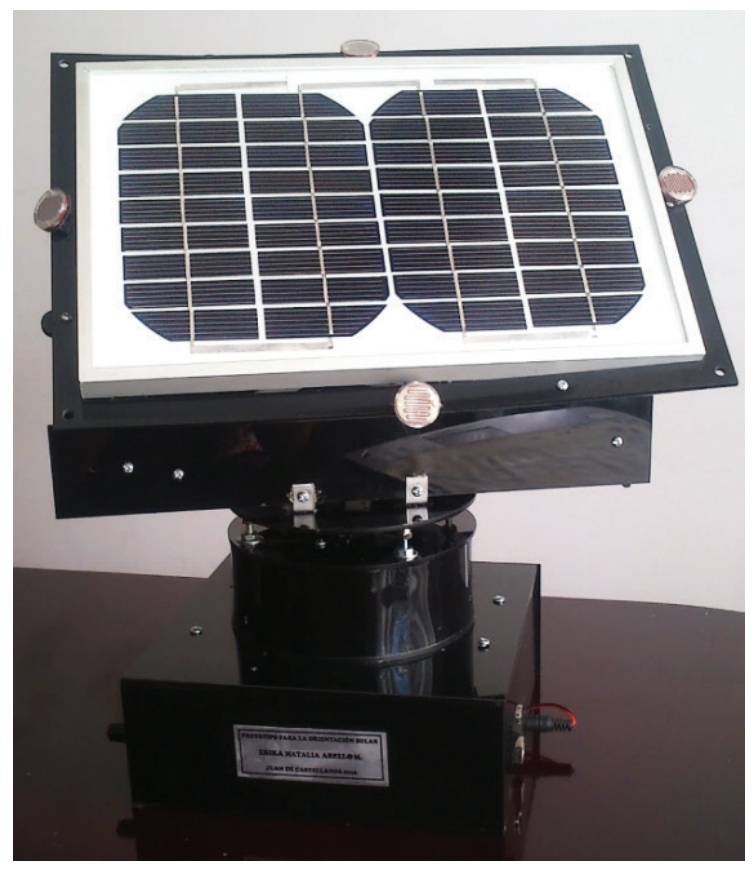

Fig. 11 Prototipo para la orientación automática de paneles solares

\section{Comparación potencia transmitida de un sistema} estático y un sistema móvil

A partir del trabajo desarrollado por Diego Mejía (Mejía, Torres y Díaz 2016) y en las pruebas tomadas del prototipo, se identificó de forma comparativa que los niveles de eficiencia y respuesta de un sistema móvil, produce un incremento en la generación de potencia respecto a un sistema estático. En la Tabla II se indican algunos de los valores de potencia registrados. 
TABLA 2.

Comparación de potencias en sistemas fijos y móviles

\begin{tabular}{|c|c|c|}
\hline Hora & Potencia Sistema Fijo & Potencia Sistema Móvil \\
\hline $6: 00$ & $0 \mathrm{~W}$ & $2.4 \mathrm{~W}$ \\
\hline $7: 00$ & $6 \mathrm{~W}$ & $10 \mathrm{~W}$ \\
\hline $8: 00$ & $20 \mathrm{~W}$ & $25 \mathrm{~W}$ \\
\hline $9: 00$ & $45 \mathrm{~W}$ & $51 \mathrm{~W}$ \\
\hline $10: 00$ & $80 \mathrm{~W}$ & $95 \mathrm{~W}$ \\
\hline $11: 00$ & $129.4 \mathrm{~W}$ & $133 \mathrm{~W}$ \\
\hline $12: 00$ & $140 \mathrm{~W}$ & $146 \mathrm{~W}$ \\
\hline $13: 00$ & $132 \mathrm{~W}$ & $136 \mathrm{~W}$ \\
\hline $14: 00$ & $82 \mathrm{~W}$ & $100 \mathrm{~W}$ \\
\hline $15: 00$ & $50 \mathrm{~W}$ & $63 \mathrm{~W}$ \\
\hline $16: 00$ & $16 \mathrm{~W}$ & $20 \mathrm{~W}$ \\
\hline $17: 00$ & $8.6 \mathrm{~W}$ & $14 \mathrm{~W}$ \\
\hline $18: 00$ & $0 \mathrm{~W}$ & $1.3 \mathrm{~W}$ \\
\hline
\end{tabular}

\section{Conclusiones y TRABAjOS FUturos}

Gracias a la investigación sobre tecnologías de posicionamiento y control para orientación de paneles solares, fue posible diseñar, ensamblar e implementar un modelo viable con los requisitos necesarios para la construcción del prototipo.

Este prototipo es de gran utilidad, ya que, al obtener datos de luminosidad a partir de sus sensores en diferentes posiciones, hace posible que se continúe en trabajos futuros la optimización de sistemas fotovoltaicos móviles.

Se logró establecer una relación directa entre la resistencia de las LDR y los lúmenes del luxómetro facilitando así el análisis del estudio lumínico.

Para optimizar y aumentar el nivel de precisión del sistema, se recomienda probar con distintos tipos de sensores de luminosidad o ampliar el número de éstos y ajustar los grados de libertad para proporcionar una elevación del panel solar, con el fin de obtener más eficiencia en la detección de la posición del sol.

Diseñar una aplicación que permita un monitoreo remoto de las señales y posición del panel fotovoltaico.

\section{RECONOCIMIENTOS}

La autora Erika Natalia Abello agradece el acompañamiento, disposición y guía por parte del Ingeniero Electrónico William Fernando Bernal, líder del grupo de investigación Biná de la Fundación Universitaria Juan de Castellanos (Fujdc) y al semillero de Investigación Tesla - en su línea de Energías Alternativas y Eficiencia Energética.

\section{REFERENCIAS}

Amarillo Rojas, M \& Trujillo Arboleda, L 2015, 'Simulación de redes de sensores inalámbricos: un modelo energético a nivel de nodo-sensor bajo las especificaciones Ieee 802.15.4tm y Zigbee', Revista Publicaciones e Investigación, 9, 14-24.

Brown, L (2015), El mundo al borde del abismo. Como evitar el declive ecológico, Ecoe-Cied, Bogotá.

Beltrán Adán, J 2007, 'Prototipo fotovoltaico con seguimiento del sol para procesos electroquímicos, Tesis de Maestría en Ciencias', Centro Nacional de Investigación y Desarrollo Tecnológico, Cuernavaca, México.

Guardado Gutiérrez, D \& Rivera Chávez, V 2012, 'Implementación de seguidor solar en dos ejes para el sistema fotovoltaico de la Escuela UES, Trabajo de graduación para optar al título de Ingeniero Electricista', Universidad de El Salvador.

Guerrero Pérez, R (2013), Replanteo y funcionamiento de instalaciones solares fotovoltáicas, IC, Antequera.

Herrera Medina, J \& Molina Palma, N 2010, 'Diseńo, análisis e implementación de un sistema de control para seguimiento solar en dos ejes, Trabajo de graduación para optar al título de Ingeniero Electricista', Universidad Don Bosco, San Salvador.

Ideam 2014, Atlas de radiación solar, ultravioleta y ozono de Colombia [Página web], consultado: 2016, http://atlas.ideam.gov. co/visorAtlasRadiacion.html

Ideam 2016, Estaciones meteorológicas [Página web], consultado: 2016, http://www.ideam.gov.co/solicitud-de-informacion. 
López Jimenéz, V 2014, 'Propuesta metodológica para el rediseńo de una red meteorológica en un sector de la región andina colombiana', Revista Publicaciones e Investigación, 8, 55-76.

López Jiménez, V, Martínez Ramos, J \& Almario Guio, D 2016, 'Análisis del indice de riesgo de la calidad del agua para consumo humano -iRca- y su relación con variables meteorológicas y ubicación geográfica para el departamento del Tolima en los años 2012-2013', Revista Publicaciones e Investigación, 10, 69-81.

Machado Toranzo, N, Lussón Cervantes, A, Leysdian Oro Carralero, L, Bonzon Henríquez, J \& Escalona Costa, O 2015, 'Segui- dor solar, optimizando el aprovechamiento de la energía solar', Ingeniería Energética, XXXVI, 190-199.

Mejía Bugallo, D, Torres Chávez, I \& Díaz Rodríguez, J 2016, 'Control Mppt aplicado a sistemas girasol', Revista Colombiana de Tecnologias de Avanzada, 1(27), 118-123.

Pallás Areny, R (2003), Sensores y acondicionadores de señal, Marcombo Boixareu Barcelona.

Upme 2014, Atlas de radiación solar de colombia [en línea], consultado: 2016,http://www.upme.gov.co/docs/atlas_radiacion_ solar/1-atlas_radiacion_solar.pdf 
